\title{
ChemComm
}

\section{Silver-catalyzed dynamic systemic resolution of $\alpha$-iminonitriles in a 1,3-dipolar cycloaddition process $\dagger$}

50,3792

Received 5th February 2014

Accepted 18th February 2014

DOI: $10.1039 / \mathrm{c} 4 \mathrm{cc} 00944 d$

www.rsc.org/chemcomm

A dynamic azomethine ylide system was established using $\mathrm{Sc}(\mathrm{OTf})_{3}$ and $\mathrm{Ag} / \mathrm{Taniaphos}$ as catalysts. The system was subsequently kinetically resolved in a tandem 1,3-dipolar cycloaddition process where the silver complex acted as both a reaction catalyst and an external selector, resulting in the formation of an exclusive pyrrolidine product in good yield and enantiopurity.

Dynamic chemistry at the constitutional level remains one of the most powerful methods for the generation and exploration of complex systems. Based on kinetically labile bonds of covalent or non-covalent nature, the spontaneous generation of systems of interconvertible adducts or complexes can be accomplished under thermodynamic control. Due to the adaptive nature of the systems, the application of internal or external pressures can lead to responsive states, where the constituents are forced to adjust to form the optimal overall arrangement. As a result of this rearrangement process, amplification of the optimal constituent(s) can take place at the expense of less favored combinations, in a primitive sense, resulting in a thermodynamically controlled evolutive process. ${ }^{1-22}$

Although reversibility is a cornerstone to achieve these effects, the resulting entities are inherently labile. Kinetically controlled steps can, however, be efficiently coupled to dynamic systems, much in the sense of common natural processes, leading to kinetic resolution of the systems, i.e. dynamic systemic resolution (DSR). If the kinetic steps are selective, complete amplification of the best fitting species will occur essentially regardless of the thermodynamic equilibrium. ${ }^{7,17,23-29}$

In addition to developing methods to explore the features and properties of complex systems per se, this resolution concept also enables efficient screening and identification of components and conditions of synthetic processes. We have, for example, more recently explored dynamic systemic resolution

Department of Chemistry, KTH - Royal Institute of Technology, Teknikringen 30, Stockholm, Sweden.E-mail: ramstrom@kth.se

$\dagger$ Electronic supplementary information (ESI) available: Experimental procedures; analytical data. See DOI: 10.1039/c4cc00944d for asymmetric heterocycle synthesis using biocatalytic action. ${ }^{30,31}$ The efficient construction of enantiopure and structurally diverse heterocycles is one of the most important tasks in synthetic medicinal chemistry. For instance, access to five-membered heterocycles, especially pyrrolidines, is highly desirable because of their wide presence in synthetic building blocks, natural products, and active pharmaceutical ingredients. ${ }^{32-34}$ Representative structures include, e.g., proline, hygrine, the antibiotic $(-)$-anisomycin, and the anti-cholinergic agent procyclidine for the treatment of parkinsonism and akathisia. Herein, we describe a dynamic systemic resolution protocol to enantioenriched pyrrolidine-2-carbonitrile structures carrying four contiguous chiral centers using a combination of transimination and ylide formation/cycloaddition catalysis (Fig. 1).

The dynamic system was generated from a sequential reversible process involving imine formation and $\mathrm{Ag}^{+}$-catalyzed azomethine ylide formation in the presence of base. $\mathrm{Ag}^{+} / \mathrm{Cu}^{+}-$ catalyzed 1,3-dipolar (Huisgen) cycloaddition is in this context considered to be one of the best strategies for the stereoselective synthesis of pyrrolidine derivatives due to the high selectivity with different chiral ligands and its atom-economical properties. ${ }^{35-40}$ Initially, generation of dynamic $\alpha$-iminonitrile systems was investigated under Lewis acidic conditions, using glycine methyl ester and four different aldehydes. The systems were thus established using 0.1 equivalents of $\mathrm{ZnBr}_{2}$ or $\mathrm{Sc}(\mathrm{OTf})_{3}$ as transimination catalysts, respectively. ${ }^{25,41} \mathrm{ZnCl}_{2}$ and $\mathrm{Zn}(\mathrm{OTf})_{2}$

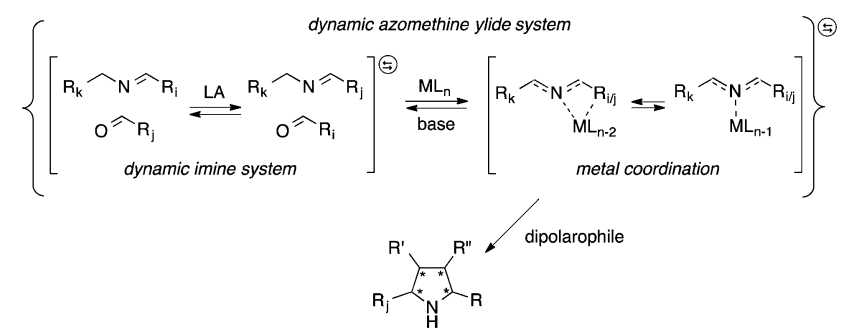

Fig. 1 Dynamic systemic resolution of metal-coordinated azomethine ylide system. 

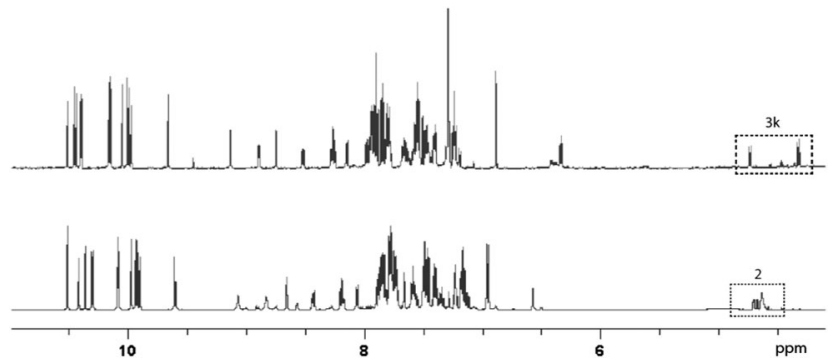

Fig. $2{ }^{1}$ H NMR spectra of the dynamic system: (bottom) at imine equilibrium; (top) after resolution.

were in this case also tested, however, resulting in lower rates. According to ${ }^{1} \mathrm{H}$ NMR spectroscopy, imine exchange was expectedly observed in both catalysts, where in this case $\mathrm{Sc}(\mathrm{OTf})_{3}$ proved more efficient than $\mathrm{ZnBr}_{2}$, promoting equilibrium formation within two hours. However, imine decomposition was also observed with both catalysts, where the imines were completely decomposed after an additional two hours reaction time in the presence of $\mathrm{Sc}(\mathrm{OTf})_{3}$. The reason for this decomposition is most likely due to polymerization of the glycine methyl ester in the presence of Lewis acid. Less reactive ethyl and isopropyl esters resulted in gradual decomposition overnight, and were also deemed not suitable for the resolution process. In order to avoid any potential polymerization, 2-aminoacetonitrile was selected instead for the process. Besides promoting ylide formation, it is worth noting that 2-cyanopyrrolidine derivatives are compounds that possess important bioactivities. ${ }^{42,43}$ The dynamic imine system was thus generated from the 2-(benzylideneamino)acetonitrile (2a), mixed with 1 equivalent each of 12 different aldehydes in the presence of 0.05 equivalents of $\mathrm{Sc}(\mathrm{OTf})_{3}$ in $\mathrm{CDCl}_{3}$. The equilibrium under these conditions was attained within 10 minutes, and no decomposition was observed even after overnight reaction. The stabilities of the different imines were also of comparable order, showing relatively isoenergetic behavior (Fig. 2). The dynamic $\alpha$-iminonitrile system was subsequently subjected to azomethine ylide formation in the presence of triethylamine in dichloromethane in a one-pot process. The Ag(I)-Taniaphos complex,${ }^{44}$ which exhibits high enantioselectivity in similar reactions, was in this case
Table 1 Screening of reaction conditions ${ }^{a}$

\begin{tabular}{lllllll}
\hline Entry & Solvent & $T\left({ }^{\circ} \mathrm{C}\right)$ & Base & $\operatorname{Yield}^{b}(\%)$ & $\mathrm{dr}^{c}(\%)$ & $\mathrm{ee}^{d}(\%)$ \\
\hline 1 & DCM & rt & TEA & 54 & $69: 31$ & 62 \\
2 & Diethyl ether & rt & TEA & 61 & $90: 10$ & 82 \\
3 & THF & rt & TEA & 72 & $78: 22$ & 89 \\
4 & THF & rt & NaOAc & 76 & $75: 25$ & 76 \\
5 & THF & 0 & TEA & 74 & $89: 11$ & 92
\end{tabular}

${ }^{a}$ Reaction conditions: compound $2 \mathrm{a}(0.12 \mathrm{mmol})$, aldehyde $\mathbf{1 b}-\mathbf{m}$ (0.12 mmol), dimethyl fumarate $(0.16 \mathrm{mmol}), \mathrm{Sc}(\mathrm{OTf})_{3}(0.006 \mathrm{mmol})$, AgOAc $(0.012 \mathrm{mmol})$, Taniaphos $(0.012 \mathrm{mmol}) .{ }^{b}$ Isolated yield. ${ }^{c}$ Deter-

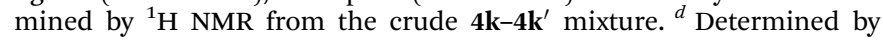
chiral HPLC (Chiralpak OJ column).

used to generate the dynamic metal coordinated azomethine ylide system and catalyze the ensuing kinetic 1,3-dipolar cycloaddition. Other ligands were also evaluated, however, resulting in low enantioselectivities. Upon addition of dimethyl fumarate as dipolarophile, pyrrolidines $\mathbf{4 k}$ (major) and $\mathbf{4} \mathbf{k}^{\prime}$ (minor) were observed as the only products in 54\% yield, $69: 31 \mathrm{dr}$ and $62 \%$ ee after 6 hours of reaction time (Scheme 1).

Since the stereoselective cycloaddition process results from the coordination between the azomethine ylide and the metalchiral ligand complex, additional coordination sites in the formed complex would potentially produce stronger binding and a more stable transition state. In the $\alpha$-iminonitrile system, both monodentate and bidentate species could be formed, of which heteroaromatic imines $\mathbf{3 b}, \mathbf{3} \mathbf{k}$ and $\mathbf{3 l}$ in principle provide two coordination sites towards $\mathrm{Ag}^{+}$, thus potentially enhancing their interactions with the catalyst and resulting in resolution preference in comparison to the other species in the system.

However, among the heteroaromatic imines, only imine $\mathbf{2 k}$ resulted in the corresponding cycloaddition products. This can be rationalized from geometry and electronic effects, favoring metal-coordinated azomethine ylide $3 \mathbf{k}$. Potential cycloaddition with compound $\mathbf{2 b}$ is thus prevented due to impaired chelation geometry with the metal center. Imines $\mathbf{2 k}$ and $\mathbf{2 l}$ share more similarity in the geometry; however, the less efficient overlapping between the $\mathrm{S}$ and $\mathrm{Ag}^{+}$orbitals makes compound $\mathbf{2 l}$ less preferred in the following 1,3-dipolar cycloaddition.

In order to improve the dynamic systemic resolution conditions, several reaction parameters were subsequently studied (Table 1).

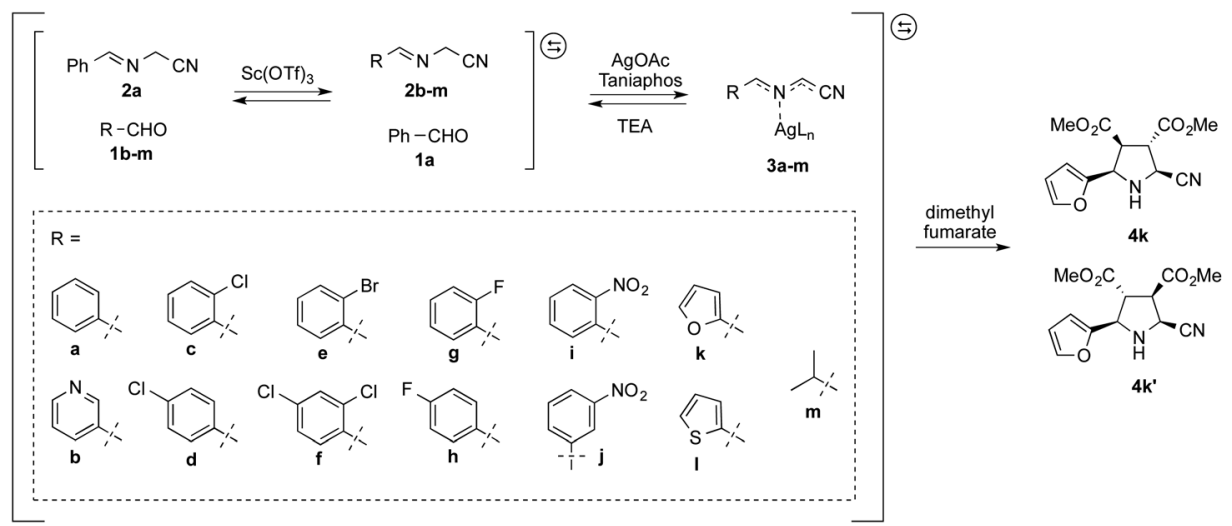

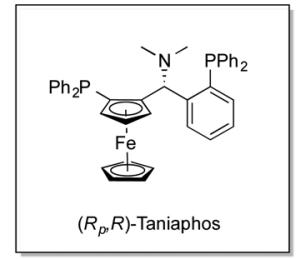

$\left(R_{p}, R\right)$-Taniaphos

Scheme 1 Silver-catalyzed asymmetric resolution of sequential dynamic system. 
Among the three solvents tested: dichloromethane (DCM), diethyl ether and THF, THF provided better yields and ees, indicating a systemic preference for more polar solvents. NaOAc and triethylamine (TEA) were evaluated as bases, of which TEA proved superior by promoting higher enantioselectivities. The effect of the temperature was also addressed. Lowering the temperature from $\mathrm{rt}$ to $0{ }^{\circ} \mathrm{C}$ significantly improved the $\mathrm{dr}$ to up to $89: 11$ while the ee also increased from $72 \%$ to $92 \%$, however, requiring a longer reaction time $(10 \mathrm{~h})$.

In summary, a sequential, dynamic metal-coordinated azomethine ylide system has been designed and developed, subsequently kinetically resolved through a coupled, silver-catalyzed 1,3-dipolar cycloaddition reaction. The silver complex acted as both a reaction catalyst and an external selector in the process, where additional coordination sites of the ylide could be used to selectively amplify the constituent with optimal properties. Exclusive formation of chiral 5-furyl-pyrrolidine products was thus obtained, in good yield, dr and ee following condition screening. This study represents the first example of a dynamic systemic resolution process of an imine system using a coupled, kinetic 1,3-dipolar cycloaddition reaction, thus expanding the reaction scope of DSR and offering a useful method for the selective synthesis of certain pyrrolidine derivatives.

This work was in part supported by the Swedish Research Council and the Royal Institute of Technology. LH thanks the China Scholarship Council for a special scholarship award.

\section{Notes and references}

1 M. Barboiu, Constitutional Dynamic Chemistry, Springer Verlag, Berlin, Heidelberg, 2012.

2 B. L. Miller, Dynamic Combinatorial Chemistry: In Drug Discovery, Bioorganic Chemistry, and Materials Science, John Wiley \& Sons, Inc., Hoboken, NJ, 2010.

3 J. N. H. Reek and S. Otto, Dynamic Combinatorial Chemistry, WileyVCH Verlag GmbH \& Co. KGaA, Weinheim, Germany, 2010.

4 M.-K. Chung, K. Severin, S. J. Lee, M. L. Waters and M. R. Gagné, Chem. Sci., 2011, 2, 744-747.

5 A. G. Santana, E. Jiménez-Moreno, A. M. Gómez, F. Corzana, C. González, G. Jiménez-Oses, J. Jiménez-Barbero and J. L. Asensio, J. Am. Chem. Soc., 2013, 135, 3347-3350.

6 M. dF Capela, N. J. Mosey, L. Xing, R. Wang and A. Petitjean, Chem.-Eur. J., 2011, 17, 4598-4612.

7 Y. Zhang, L. Hu and O. Ramström, Chem. Commun., 2013, 49, 1805-1807.

8 N. Roy and J.-M. Lehn, Chem.-Asian J., 2011, 6, 2419-2425.

9 Z. Fang, W. He, X. Li, Z. Li, B. Chen, P. Ouyang and K. Guo, Bioorg. Med. Chem. Lett., 2013, 23, 5174-5177.

10 P. Reeh and J. de Mendoza, Chem.-Eur. J., 2013, 19, 5259-5262.

11 A. Martínez-Castañeda, H. Rodríguez-Solla, C. Concellón and V. del Amo, Org. Biomol. Chem., 2012, 10, 1976-1981.

12 Y. Dong, X. Mao, X. Jiang, J. Hou, Y. Cheng and C. Zhu, Chem. Commun., 2011, 47, 9450-9452.
13 A. M. Belenguer, T. Friščić, G. M. Day and J. K. M. Sanders, Chem. Sci., 2011, 2, 696.

14 F. Mansfeld, H. Au-Yeung, J. Sanders and S. Otto, J. Syst. Chem., 2010, 1, 12.

15 B. Buchsnée Levrand, G. Godin, A. Trachsel, J.-Y. de Saint Laumer, J.-M. Lehn and A. Herrmann, Eur. J. Org. Chem., 2011, 681-695.

16 M. Rauschenberg, S. Bomke, U. Karst and B. J. Ravoo, Angew. Chem., Int. Ed., 2010, 49, 7340-7345.

17 R. Sundell, M. Turcu and L. Kanerva, Curr. Org. Chem., 2013, 17, 672-681.

18 S. Ito, K. Ono and N. Iwasawa, J. Am. Chem. Soc., 2012, 134, 13962-13965.

19 L. Marin, B. Simionescu and M. Barboiu, Chem. Commun., 2012, 48, $8778-8780$

20 J. W. Sadownik and D. Philp, Angew. Chem., Int. Ed., 2008, 47, 9965-9970.

21 T. K. Ronson, S. Zarra, S. P. Black and J. R. Nitschke, Chem. Commun., 2013, 49, 2476-2490.

22 K. Osowska and O. Miljanić, Synlett, 2011, 1643-1648.

23 M. Sakulsombat, Y. Zhang and O. Ramström, Top. Curr. Chem., 2012, 322, 55-86.

24 Y. Zhang, L. Hu and O. Ramström, in Supramolecular Systems in Biomedical Fields, ed. H.-J. Schneider, RSC Publishing, Cambridge, UK, 2013, pp. 397-418.

25 P. Vongvilai and O. Ramström, J. Am. Chem. Soc., 2009, 131, 14419-14425.

26 Y. Zhang, M. Angelin, R. Larsson, A. Albers, A. Simons and O. Ramström, Chem. Commun., 2010, 46, 8457-8459.

27 R. Caraballo, H. Dong, J. P. Ribeiro, J. Jiménez-Barbero and O. Ramström, Angew. Chem., Int. Ed., 2010, 49, 589-593.

28 P. Vongvilai, M. Angelin, R. Larsson and O. Ramström, Angew. Chem., Int. Ed., 2007, 46, 948-950.

29 M. Sakulsombat, P. Vongvilai and O. Ramström, Org. Biomol. Chem., 2011, 9, 1112-1117.

30 M. Sakulsombat, Y. Zhang and O. Ramström, Chem.-Eur. J., 2012, 18, 6129-6132.

31 L. Hu, F. Schaufelberger, Y. Zhang and O. Ramström, Chem. Commun., 2013, 49, 10376-10378.

32 J. P. Michael, Nat. Prod. Rep., 2008, 25, 139-165.

33 D. Enders and C. Thiebes, Pure Appl. Chem., 2001, 73, 573-578.

34 S. G. Pyne, A. S. Davis, N. J. Gates, J. P. Hartley, K. B. Lindsay, T. Machan and M. Tang, Synlett, 2004, 2670-2680.

35 L. M. Stanley and M. P. Sibi, Chem. Rev., 2008, 108, 2887-2902.

36 M. Naodovic and H. Yamamoto, Chem. Rev., 2008, 108, 3132-3148.

37 H. Y. Kim, H.-J. Shih, W. E. Knabe and K. Oh, Angew. Chem., Int. Ed., 2009, 48, 7420-7423.

38 A. López-Pérez, J. Adrio and J. C. Carretero, J. Am. Chem. Soc., 2008, 130, 10084-10085.

39 A. López-Pérez, J. Adrio and J. C. Carretero, Angew. Chem., Int. Ed., 2009, 48, 340-343.

40 X.-H. Chen, W.-Q. Zhang and L.-Z. Gong, J. Am. Chem. Soc., 2008, 130, 5652-5653.

41 N. Giuseppone, J.-L. Schmitt, E. Schwartz and J.-M. Lehn, J. Am. Chem. Soc., 2005, 127, 5528-5539.

42 O. Seitz, T. Kondo, T. Nekado, I. Sugimoto, K. Ochi, S. Takai, A. Kinoshita, A. Hatayama, S. Yamamoto, K. Kawabata, H. Nakai and M. Toda, Bioorg. Med. Chem., 2008, 16, 190-208.

43 S. W. Wright, M. J. Ammirati, K. M. Andrews, A. M. Brodeur, D. E. Danley, S. D. Doran, J. S. Lillquist, L. D. McClure, R. K. McPherson, S. J. Orena, J. C. Parker, J. Polivkova, X. Qiu, W. C. Soeller, C. B. Soglia, J. L. Treadway, M. A. VanVolkenburg, H. Wang, D. C. Wilder and T. V Olson, J. Med. Chem., 2006, 49, 3068-3076.

44 R. Robles-Machín, I. Alonso, J. Adrio and J. C. Carretero, Chem.-Eur. J., 2010, 16, 5286-5291. 